\title{
Rethinking interdisciplinarity: Proposing a multilayered model
}

\author{
Shahram Yazdani ${ }^{1}$, Maryam Hajiahmadi*1 $\mathbb{D}$, Sareh Shakerian ${ }^{2}$ \\ Received: 19 Mar 2019 \\ Published: 10 Sep 2020
}

\section{Abstract}

Background: One of the efforts that solve complex real-world problems is to use an interdisciplinary approach. However, the contradictions among different disciplines and the absence of a theoretical model add to the difficulty of interdisciplinary activities. When interdisciplinary researchers face a complex problem, they need to integrate several different disciplines. It seems the first step in interdisciplinary activities is to have a theoretical model to understand and examine by what content and method the processes of integrating and crossing the disciplinary boundaries are done. The purpose of this study is to propose a new theoretical model for interdisciplinary development. It also show that a multilayered model can be formed based on the boundaries of scientific disciplines.

Methods: In this study, the critical review strategy of Carnwell and Daly has been used to synthesize the theoretical model using online databases. The method consists of 5 stages: defining the scope of the review, identifying the sources of relevant information, reviewing the literature, writing the review, and applying the literature to the proposed study.

Results: The results of this study showed for interdisciplinarity, the artificial boundaries of disciplines must be broken. Therefore, we first began to explain the disciplinary boundaries in 13 levels, then, we proposed interdisciplinary development by examining the views of the experts in this field and with the synthesis opinion of researchers of this study on each of the disciplinary boundaries of the multilayered model.

Conclusion: This model can effectively support interdisciplinary programs and can bring the goals of interdisciplinary programs closer to practical reality.

Keywords: Disciplinarity, Interdisciplinarity, Theoretical model, Critical review, Model

Conflicts of Interest: None declared

Funding: This work was part of a PhD dissertation and supported by Shahid Beheshti University of Medical Sciences (Grant number: 66005836).

\section{*This work has been published under CC BY-NC-SA 1.0 license.}

Copyright $\odot$ Iran University of Medical Sciences

Cite this article as: Yazdani Sh, Hajiahmadi M, Shakerian S. Rethinking interdisciplinarity: Proposing a multilayered model. Med J Islam Repub Iran. 2020 (10 Sep);34:118. https://doi.org/10.47176/mjiri.34.118

\section{Introduction}

The $21^{\text {st }}$ century will be known as a century in which the combination of disciplines becomes evident. Also, it will be recognized as a century of partnership, cooperation, and interdisciplinarity and as a century in which important questions about health, disease, actions, and recovery can-

Corresponding author: Maryam Hajiahmadi, m.hajiahmadi@sbmu.ac.ir

1. Virtual School of Medical Education and Management, Shahid Beheshti University of Medical Sciences, Tehran, Iran

2. Community Based Educational Health System Department, Shahid Beheshti University of Medical Sciences, Tehran, Iran not be solved in terms of discipline or science. In other words, one type of discipline cannot meet the environmental needs and challenges (1).

Aboelela et al argued that "Merely bringing together different scholars from diverse disciplines with different aca-

\section{$\uparrow$ What is "already known" in this topic:}

According to the literature review, authors believe having a theoretical model for implementing interdisciplinary activities is essential. The review has confirmed the lack of a theoretical model or structure for implementing interdisciplinary activities.

\section{$\rightarrow$ What this article adds:}

Based on the views of the experts in this field and the synthesis opinion of researchers in this study, we have outlined and suggested a "multilayered model" of interdisciplinary development. It seems this model can operationally support interdisciplinary programs and bring the objectives of interdisciplinary programs closer to practical reality. 
demic degrees is not enough for interdisciplinarity of research." In his view, for a desirable interdisciplinary work, it is necessary to integrate disciplines, collect, analyze data, and make a conclusion in the research process regarding the conceptual framework development (2).

Also, according to the National Academy of Sciences, studies are interdisciplinary when the work done is not just to tie the 2 disciplines together and to produce a new product, but methods and ideas must be merged and combined in it (2).

Pirrie et al argue interdisciplinary researchers are academicians working across their common disciplinary boundaries and even if they keep their focus on their disciplinary boundaries, they still benefit from the concepts and techniques of other disciplines. In any case, since they are working across the disciplinary boundaries, their work is considered to be interdisciplinary $(3,4)$.

Rogers et al express concern about the integration of disciplines. They believe that "many epistemological limitations make it difficult to achieve interdisciplinarity." An example of such constraints is diverse analytic units, different opinions about reality, expectations, criteria, and value judgments (5).

According to Joseph Kockelmans, our knowledge system has dangerous epistemological boundaries and classification, because each discipline has produced its own general framework, theories, and methods $(6,7)$.

The distinctive and complex differences and characteristics among different disciplines make the situation difficult for interdisciplinary actors. When interdisciplinary researchers encounter a complex problem, it may be necessary to integrate several opposing disciplines to examine the issue. Different disciplines have different boundaries that can cause problems when combined. By reviewing the interdisciplinary literature, we faced a challenge in this area: the lack of a theoretical model for interdisciplinary development, as no solution has been suggested yet. Our suggestion in this study is to design and synthesize a new theoretical model for interdisciplinary development to help actors in the field of interdisciplinarity to overcome the problems caused by the lack of a theoretical model by providing a new route.

\section{Methods}

In this study, the critical review method proposed by Carnwell and Daly has been used to present and synthesize a conceptual model of interdisciplinarity, which includes the following stages (8).

1. Defining the scope of the review;

2. Identifying the source of relevant information;

3. Reviewing the literature;

4. Writing the review;

5. Applying the literature to the proposed study;

As Carnwell and Daly (8) suggested in the "strategies for the construction of a critical review of the literature," we first defined the scope of the review. According to the researchers' belief that interdisciplinary ideas have been constructed based on disciplines, our review included 2 parts. The first was the review of the disciplinary concepts and the second to review interdisciplinary literature. A systematic search for texts was done in databases and related websites. After having screened and found related texts, we reviewed the original text of the articles to understand why and how to study. The results of this review both made us aware of the recent state of knowledge in the field of interdisciplinarity and clarified the existing gaps in theoretical knowledge as well as the methodological limitations in developing a relationship among disciplines. Ultimately, efforts in reviewing the literature, and researchers' opinions have led to the synthesis and presentation of a theoretical model of disciplinarity and interdisciplinarity for interdisciplinary actors.

\section{The stages of critical review are as follow:}

1. Defining the scope of the review: In this study, it was aimed to review the views of scholars on the criteria of scientific discipline to extract the criteria that have the most similarity with the discipline to propose a theoretical model for interdisciplinary development with the recognition of the disciplinary boundaries.

2. Identifying the source of relevant information: Given that this study aimed to achieve a theoretical model for interdisciplinary development, we first need to consider the criteria of the scientific disciplines for the precise identification of this model. Thus, we first critically reviewed the disciplinary criteria and then proposed a theoretical model. The resources were identified through a systematic and comprehensive search of texts using the Google Scholar, ERIC, PubMed, Web of science, Embase for relevant publications up to 2016.

The keywords used in our search strategy were "discipline boundaries", "discipline definitions", "discipline discrimination", "discipline framework".

Then, the key words "interdisciplinarity theory", "interdisciplinarity model", and "interdisciplinarity framework" were used separately for each boundary we had defined. Time of publication was not a concern. The reviewing process was repeated to ensure lack of exclusion of any single article unintentionally. In the reviewing process, duplicate studies were excluded as well as those whose title or abstract were irrelevant to the study subject. Also, some articles were also excluded due to lack of access to their full-text.

Article with the following criteria were included: (1) articles published in English and Persian; and (2) studies with interdisciplinarity approaches. If an article provided a theory which was out of our defined boundaries or was a case report, it was excluded.

3. Reviewing the literature: At this stage, the researcher briefly examined the abstract and original text of the articles to understand why and how those studies were performed. The primary screening was done by examining the titles to remove the unrelated sources. Then, the abstracts and texts of the articles were studied and the articles which had the criteria and characteristics of the discipline were selected and their full texts were studied. Research papers or articles in languages other than Persian and English were removed at this stage.

The articles and texts used in the study were prioritized according to their relevance to the research objectives and 
the rate of citations. One of the limitations of this study was the use of English articles and inaccessibility to some databases.

4. Writing the review: The results of this study were prepared in 2 parts. The first part focuses on explaining the boundaries of the academic discipline and the second introduces the multilayered model of interdisciplinary development.

5. Applying the literature to the proposed study: Finally, the review of the literature, along with the opinions and synthesis of researchers, has led to the synthesis and presentation of a multilayered model for interdisciplinary development.

\section{Results}

Stage 1. A multilayered model of discipline: The review in the first stage of the study showed interdisciplinary authors believe the focus of interdisciplinary activities is on the integration of different disciplines $(7,9,10)$.

Also, the importance of the position and role of the scientific disciplines in interdisciplinary activities, identifying, and explaining the disciplinary boundaries help identify the aspects separating disciplines and establish a model for interdisciplinary activities. Therefore, before breaking boundaries between different disciplines, it is necessary to envision a clear theoretical framework or a model of the disciplinary boundaries.

Various disciplinary boundaries or levels have been defined and explained by Cohen (1970), Fouco (1980), Bashar (1989), Booker (2003), Zeustock (2007), Davies and Dolin (2007), Krishnan (2009), Ken Fuchsman ( 2009), Jo Morgan (2002), Howard Gardner( 2000), Klein (1990), Bunge (1983), and David Rousseau et al (2016) (1-10). In this study a multilayer model of scientific disciplines is presented in the form of pyramids through reviewing, integrating, and generalizing view points and descriptions of disciplines. In this pyramid-shaped model, base (or substructure) boundaries are placed at the bottom of the model and the superstructures at the top. This model includes 13 main layers and 37 sublayers. Each layer shows an area of scientific discipline. The definitions of each main or sublayer are as follow (Fig. 1):

\section{1) A philosophical background}

i. Ontological assumptions: To our knowledge, it is the phenomenon for which thinkers within the scope of a discipline consider an existence, and it is the subject of recognition and intervention in that discipline.

ii. Epistemological assumptions: Any knowledge which can be assumed by the thinkers of that discipline about the phenomena or subject matter in one discipline; eg, the explanation, description, definition, and justification.

iii. Methodological assumptions: Any form of inquiry by scholars of discipline is considered comprehensive for acquiring knowledge.

iv. Semantic assumptions: The range of semantic meanings and labels provided by scholars of a discipline on the phenomena or the subject matter of the discipline and the relationships among them.

v. Axiology: Anything assumed by scholars as a desirable end for the phenomenon or subject matter in a discipline is considered to have a cognitive value. The effort of scientific activities in one discipline will ultimately lead to a desirable goal.

\section{2) Culture}

i. Customs/ social behavior: Set of rules or traditions that govern the relationships among individuals in the

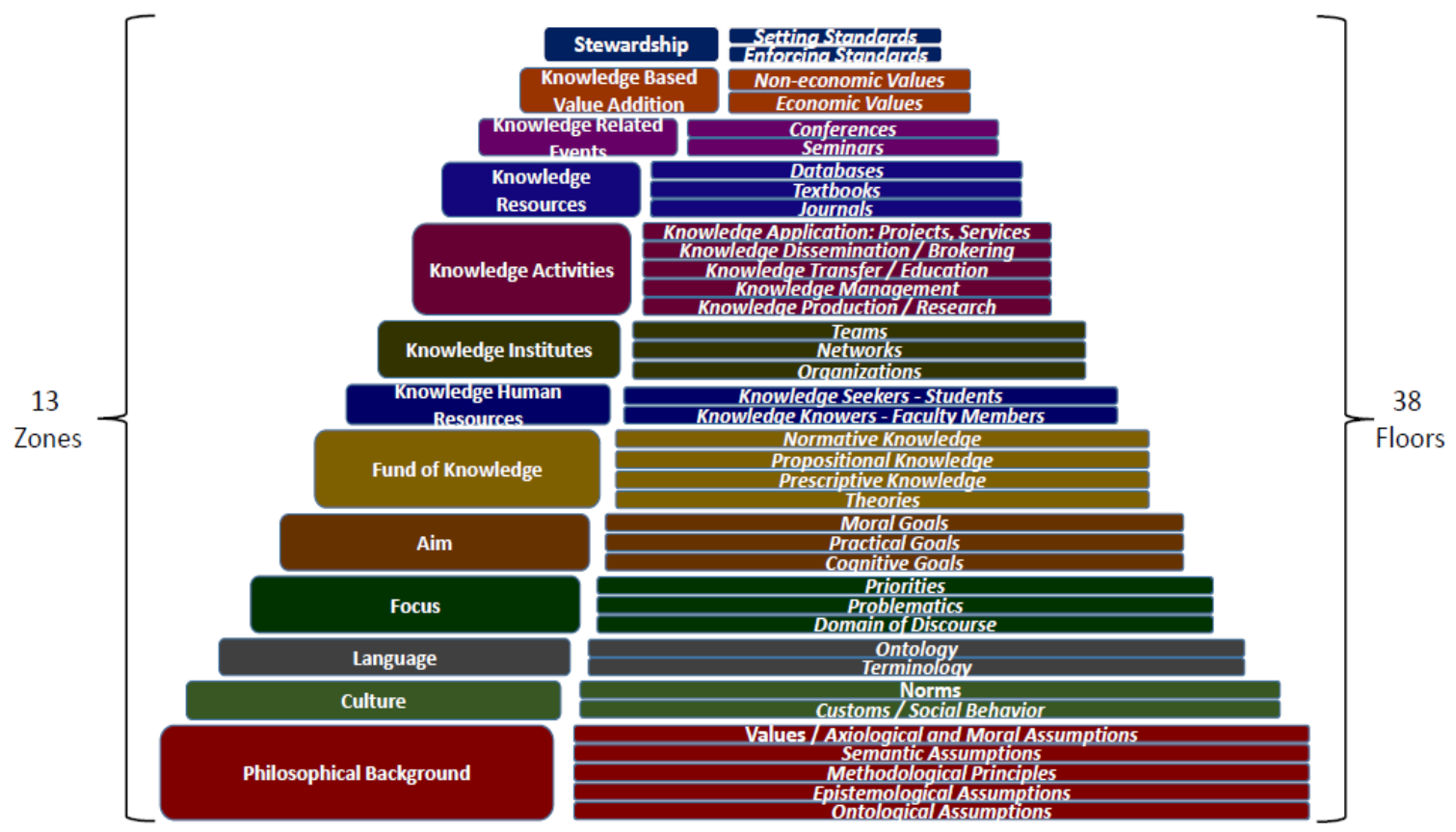

Fig. 1. The multilayered model of boundaries of a scientific discipline 
scientific community of a discipline.

ii. Norms: A set of values that are abstracted from the normative behavior of scientific actors within the scope of a single discipline.

\section{3) Language}

i. Terminology: The terms used to make a scientific relationship among scientific actors within the scope of a discipline.

ii. Anthology: The semantic system that dominates the collection of scientific vocabulary in one discipline.

\section{4) Focus}

i. The domain of discourse: A set of scientific subjects that is the main concern of the discipline and it is the main subject of thinking, effort, and interaction.

ii. Problematics: A set of main issues that are the subject of the researchers in a discipline, the desired integrity in the phenomenon, or the subject of a discipline are achieved by solving these problems.

iii. Priorities: Important issues that are determined to identify goal-setting and intervention on the phenomena of a given discipline, and they are the basis for the research orientation in a discipline.

\section{5) The aim}

i. Cognitive goals: A set of goals that are determined in relation to the recognition of the actual state of phenomena of the subject of a discipline. The realization of these goals leads to the full knowledge of the phenomena and their behaviors in the natural state.

ii. Practical goals: A set of goals that are determined for potential intervention on the phenomenon to achieve the desired condition of the phenomenon.

iii. Moral goals: A set of goals that are determined by considering cognitive value assumptions regarding the desired state of phenomena discussed in a discipline.

\section{6) Fund of knowledge}

i. Theories: A set of consonant propositions (regular, clear, and organized) that are used to describe, justify, and predict the behavior of a phenomenon.

ii. Descriptive knowledge: A form of knowledge that is about the behavior of phenomena in its real framework.

iii. Prescriptive knowledge: A form of knowledge that is used to intervene and change the behavior of a phenomenon in a discipline toward desirable behavior.

iv. The contractual knowledge: The desirable condition of a phenomenon in a discipline.

\section{7) Knowledge human resources}

i. Faculty members: Individuals who are able to understand deeply the phenomena and problem-solving specific to that discipline using a collection of codified knowledge and acquisition of tactic knowledge.

ii. Knowledge seekers-students: They are ones who acquire knowledge and experience in each discipline and advance from the marginal position to the pivotal position in a discipline through the acquisition of academic qualifications and the formation of scientific identity over time.

\section{8) Knowledge institutions}

i. Organizations: arrangements, formal and observational frameworks by which scientific actors in a discipline deal with scientific activities.

ii. The communication network: frameworks that link the broad range of scientific actors in a discipline to different scientific organizations.

iii. Teams: A set of scientific actors in a discipline that deals with scientific coordination activities with a common scientific objective.

\section{9) Knowledge activities}

i The production of knowledge/research: A form of scientific activity whose purpose is to add the current knowledge about the phenomena discussed in a discipline.

ii. Knowledge management: All activities in a discipline to summarize, integrate, and localize knowledge. The purpose of knowledge management is to acquire different knowledge products that can be used by knowledge users in a discipline.

iii. The knowledge transfer/education: The process of knowledge transfer and the development of qualifications and the formation of scientific identity in the learners of discipline, and it is usually managed by the scientists in that field.

iv. The knowledge distribution/marketing: The process of publishing and transferring, ensuring access, increasing the understanding, and facilitating the use of knowledge by knowledge users in a discipline.

v. Knowledge application: Projects, services, and knowledge crystallization suggested in a discipline in the form of services, provided services, or manufactured products.

\section{0) Knowledge resources}

i. Periodical publications that are produced in any discipline for the transfer of foreground knowledge and published by researchers.

ii. Textbooks: Publications that are produced to transfer background knowledge in a discipline and published by researchers.

iii. A database: A collection of structured information that is generated for archiving and indexing knowledge in a discipline. It provides the knowledge for the scientific actors through search engines.

\section{1) Knowledge related events}

i. Seminar: A kind of academic education, which is provided by an academic institution. Participants will discuss a specific issue in a discipline to get more and deeper information about it and obtain new results.

ii. The conference: A variety of gatherings that are organized with official agenda for discussion, negotiation, consultation, or exchange of information to find a solution and settle a specific problem in a discipline.

\section{2) Knowledge-based value addition}

i. Economic values: The financial added value through the provision of services, knowledge, or the sale of knowledge products in a particular discipline. 
ii. Nonfinancial values: They are achieved through the use of knowledge generated in a discipline at the community level, such as justice and sustainability development.

\section{3) Stewardship}

i. Implementing standards: To ensure the integrity of individuals, functions, and the accountability of the larger scientific and social community, each discipline requires institutions to evaluate different functions in accordance with standards by organizing, monitoring, and accrediting them to achieve the goals of a discipline.

ii. Setting a standard: An institution or position that is responsible for policymaking, prioritization, strategic planning, and regulation to determine the quality and desirable characteristics of graduates of each discipline to create new knowledge, protect and transfer ideas through writing, teaching, and use of discipline.

Stage 2. Proposing a multilayered model for interdisciplinarity

Our goal is to formulate a theoretical model framework for interdisciplinary activities. Thus, it is essential to deeply understand based on what model individuals with distinct disciplinary boundaries come up with consensus or consider the opinions of their colleagues for a metadisciplinary problem-solving.

Although the implementation of the interdisciplinary plan is rare in the form of a theoretical framework, the results of some studies indicate philosophers, writers, and experts in this field have focused on interdisciplinary distinctions and considered a theoretical model necessary to build a more comprehensive and common view.

A multilayered model, which is based on bridging among disciplinary boundaries, is a good model to understand associations between different disciplines for interdisciplinary activities. This model is presented in Figure 2.

\section{1) Philosophical Background}

A study was conducted on papers and researches of Schmidt (13), Little (14), Miller et al (15), Miller and Mansilla (16). Although they showed in interdisciplinary programs philosophy is effective in analyzing and classifying interdisciplinarity, they did not define the interaction and integration of philosophical assumptions of the discipline clearly. To our knowledge, ontological assumptions, any knowledge of phenomena, methodological principles, semantic assumptions, and the assumption of the cognitive value that are the philosophical presuppositions of a discipline must be understood and integrated into interdisciplinarity. In other words, understanding the philosophical presuppositions in each of the disciplines is an essential prerequisite for understanding and developing interdisciplinarity.

\section{2) Culture}

Ylijoki (17), Wesselink (18), Klein (19), in their studies, stated normative choices play an important role in interdisciplinary research. In general, they have studied cultural differences in interdisciplinary research. Although we agree with their statements, we emphasize interdisciplinary actors should make changes in the behaviors and values that govern relationships between them and cross characteristics of the cultural boundary, including customs/ social behavior, and disciplinary norms, to create a new cultural boundary.

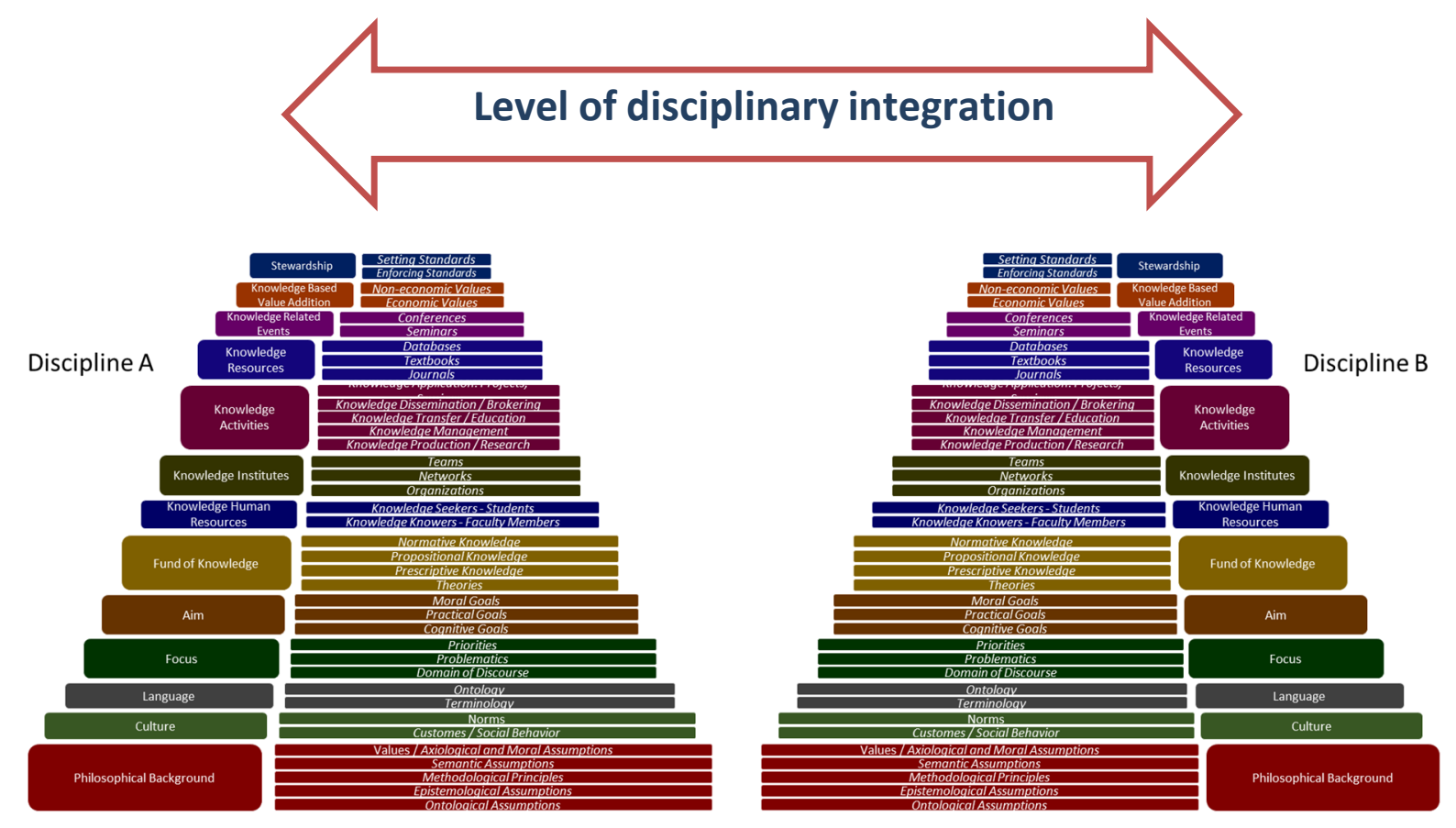

Fig. 2. A multilayered model for interdisciplinarity 


\section{3) Language}

Various authors such as Wear (20), McCallin (21), Monteiro and Keating (22), Schnieder and Wegener (23) referred to the specific language of a scientific discipline and believe each scientific discipline develops its linguistic requirements. Different disciplines need a common language to use their findings so that the readers of various disciplines can understand them. Disciplines are often written in their own language and terminology, which can be incomprehensible or even misleading for unfamiliar readers. To our knowledge, each scientific discipline creates knowledge and forms a theoretical system. In this theoretical system terminology and ontology are used for scientific communication among scientific actors within the scope of the discipline. Undoubtedly, the development of linguistic capabilities, linguistic communication, and common language is essential in interdisciplinary interactions, and the stronger and deeper the communication, the greater the possibility of integrating various disciplines.

\section{4) Focus}

Vosskamp et al (24), noticed 5 points related to the meaning and purpose of interdisciplinarity. First, interdisciplinary collaboration creates new opportunities for new and constructive combinations. Second, the interdisciplinary focus is on the solutions of major issues, such as peace or the environment. Third, interdisciplinary activities focus on the unity of disciplines. Fourth, nowadays, the unification of scientific disciplines reflects itself as science. Fifth, research on discipline and interdisciplinarity is a key factor in the production of knowledge. These issues are important for understanding the structure and the way interdisciplinary activities are performed, as the concern of solving complex problems in the world needs to integrate topics of several scientific disciplines. Problems that occur simultaneously in some scientific disciplines and do not fall within the specific framework of a scientific discipline should be in line with the goals of interdisciplinarity. On the other hand, integrated research is increasingly used to solve widespread and complex social problems. Their focus is on crossing disciplinary boundaries to generate new knowledge and theory and to achieve a common research goal. It means the research question should be defined and prioritized commonly and the answer can be achieved through the knowledge integration of different disciplines.

\section{5) Aim}

Fischer (25), Stein (26), and Mansilla (10) supported the complex and hierarchical qualifications of interdisciplinary activities, because they are problem-centered and encounter social values and virtues proportional to requirements of cognitive and social knowledge. Briefly, considering the criteria provided by each discipline and demonstrating the existence of cognitive, practical, and moral goals in each discipline, 3 characteristics of the aim boundary should be used in the integration of scientific disciplines: first, cognitive goals should be used to understand the actual state of phenomena, full recognition of phenomena, and their behavior in a natural state. Sec- ond, practical goals are a set of goals for the potential intervention on the phenomenon to achieve a desirable situation and an end. Third, moral goals are a set of goals that take into account cognitive value assumptions related to the desired condition of phenomena.

\section{6) Fund of Knowledge}

Van den Besselaar and Heimeriks (27), Sawa (28), and Aram (29) offer a completely different organization of knowledge acquisition. They believe as society needs more applied knowledge, the combination and integration of knowledge of various scientific disciplines are inevitable. In other words, the scientific system is not only quantitatively growing, but it is also changing in the structure and function of the research position, the patterns of participation, and the objectives of scientific investment, which is done in the broad range of communication and comparison of ideas in a variety of disciplines. However, they did not specifically refer to the fund of knowledge boundary and its main characteristics in integration of scientific disciplines.

To our knowledge, each scientific discipline is a branch of knowledge with consistent relevant theories. Each discipline knowledge is divided into descriptive, prescriptive, and applied knowledge according to their goals, which answers the questions of that discipline. Data, information, and knowledge of each discipline are stored at a specific location. For example, medical researchers refer to Medline for information and knowledge they need, or researchers refer to Eric to search for an education-based knowledge. Their goal is to manage knowledge, communicate with experts and experienced individuals who need specific knowledge.

Interdisciplinarity does not limit itself to a scientific discipline to explain a phenomenon, intervention, behavioral change of the phenomenon, or determine the desired state of a phenomenon, but it goes beyond academic boundaries. Merged experiences usually occur when actors integrate theories, data, information, knowledge, 2or more disciplines, or a knowledge institution to increase basic understanding, produce new knowledge, or solve problems. Therefore, to search for knowledge stored in interdisciplinarity, we cannot limit ourselves to a single knowledge discipline and we need the same knowledge storage. For example: Due to the unimaginable increase in content production and the same knowledge storage, Google is the top facilitating and helpful search engine for extraction and display of the required information and knowledge from databases and sites.

\section{7) Knowledge Human Resources}

Millar (30), Lattuca (31), Jakab and Lukic (32) emphasize interdisciplinary learning and teaching are to prevent teachers and students from the separation of disciplines and target a combined knowledge. To our knowledge, participants, eg, faculty members and students, should go beyond academic boundaries in interdisciplinarity to find a way for a deep understanding of phenomena, creation, identification, and acquisition of knowledge from real experience. Integrated experiences will occur when learn- 
ers face problems in real, wide, and unknown world; thus, they should cross different areas of past knowledge and enjoy various research methods.

The integrated learning experiences of students and faculty members are taking place in a complex situation outside of the discipline. Since these people are in different social positions, they look at the situation from different angles and understand different experiences. The challenge of integrated learning leads to the production of new knowledge with the goal of a comprehensive and deep understanding of complex phenomena and issues.

\section{8) Knowledge Institutions}

Palmer et al (33), Klein (34) Vosskamp et al (24), and Berte (35) confirmed numerous challenges for the application of interdisciplinarity in disciplinary institutions. Interdisciplinary activities are in contrast with traditional universities and research centers, as they bring together the disciplines and enable the knowledge to cross the disciplinary boundaries. Thus, they have addressed the reorganization of institutional space.

To our knowledge, the strategy of the traditional university structure should be changed. The academic spaces should be ready to integrate interdisciplinary principles. Interdisciplinary schools and departments should also be established. That is, instead of producing a disciplinebased knowledge, interdisciplinary knowledge should be produced. This reform will solve academic or institutional structural challenges, communication, and team networks. Interdisciplinarity can be obtained with the creation of a shared vision among individuals and interactions between the formal and informal institutions in discipline-based universities.

\section{9) Knowledge activities}

The literature and writers such as Prager et al (36), Burger et al (37), Jacobs and Frickel (38), O'Rourke et al (39), and Johnson (40) have identified interdisciplinary activities in teaching, education, research, and professional activities.

As mentioned earlier, activities of research knowledge production, knowledge management, knowledge transfer, knowledge distribution, and the use of knowledge are dependent on scientific disciplines. Thus, interdisciplinary actors, in their various activities integrate knowledge and thinking methods into the disciplinary boundaries to reach a common understanding with the exchange and synthesis of knowledge and solve complex problems.

\section{0) Knowledge Resources}

According to Klein $(34,41)$, JAKAB and LUKIC (32), the richest source for interdisciplinary education and research is its enormous literature. This literature shows a wide range of interdisciplinary activities as well as its theoretical and ideological positions. Interdisciplinary activities in the field of applied research are distributed with regard to social, ethical, and political dimensions of a subject.

We believe the inactive and disciplinary mechanisms, transfer, and distribution of knowledge resulting from interdisciplinary research (eg, publishing articles in domestic and foreign journals or presenting them at domestic and foreign conferences, seminars, and congresses) do not have an effect on the active involvement of interdisciplinary actors. On the other hand, interdisciplinary literature is produced through its goal, which is combination of the knowledge of different disciplines to solve complex social problems. Therefore, a strategy should be devised to transfer interdisciplinary knowledge, provide its interactive distribution among researchers and different reader,s and cause changes in behavior and attitude.

\section{1) Knowledge-related events}

Berger et al (37) and Frost and Jean (42) are trying to arrange seminars or conferences to provide cross border solutions to the real-world issues of the universities and to discuss and develop relationships among different disciplines.

Nowadays, universities are organizing different seminars to deepen specialized disciplines so that the participants can discuss a particular issue in a specific discipline and obtain more and deeper information and new results. They may also try to hold conferences for negotiation, consultation, or exchange of information to find solutions and solve specific problems in a discipline.

Interdisciplinary seminars and conferences seem to be a valuable effort to change the attitude toward their discipline and other disciplines. The conference predisposes to discourse, pathology, and ultimately finds the right strategies and models for solution of real problems and development at the university.

\section{2) Knowledge-based value addition}

Andre and Frochot (43), while discussing the difficulties in evaluations of interdisciplinary research, believe recent developments in innovation reveal the complex, numerous, and opportunistic connections among systems that have gone beyond single disciplines. Social responsibility and attention to social needs and problem-solving emphasize interdisciplinary research, which should have a comprehensive view and bring about sustainable development.

Nowadays, with the expansion of the dimensions and speed of social changes and the development of the field of science, interdisciplinary activities are accelerated and the necessity of the connection among scientific disciplines has become inevitable. Effective use of professional or academic teams from several different disciplines can help find new solutions to the complex problems, social and economic questions of natural and political resources and reach economic and nonfinancial values, such as justice and sustainable development.

\section{3) Stewardship}

Holley (44) and Lyall et al (45) have proposed strategies for interdisciplinary actors to be successful in their performance. In other words, they are obliged to observe the rules and regulations of knowledge. To our knowledge, stewardship is to maintain the integrity of a discipline. Therefore, to ensure the integrity of individuals, functions, and the accountability of the larger scientific and social 
community, each discipline usually requires institutions that are responsible for policymaking, prioritization, strategic planning, function organization, monitoring, and accreditation in discipline. Stewardship is a function through which one can achieve the goals and ideals of a discipline. Examples of these institutions are scientific boards in disciplines with educational function and scientific associations whose function is research. An accreditation institution in a discipline is responsible for issuing a work license or a document certificate.

Therefore, in interdisciplinarity we will need changes in adjusting and implementing disciplinary standards.

\section{Discussion}

By reviewing the literature we found a great deal of effort and interest in theorizing interdisciplinarity, classification, and differentiation of words; for example, multidisciplinarity, interdisciplinarity, etc. This existing literature does not respond to the new questions of interdisciplinary actors, how the disciplines are integrated, or to what degree different levels of interdisciplinarity show our knowledge gap in this regard.

Ghnassia and Seabury stated, "we need a structure to integrate interdisciplinarity, not merely thinking," which confirms this gap, and there is very little information (46).

Although the implementation of the interdisciplinarity plan is rare in the form of a theoretical framework, the results of some studies indicate that philosophers, writers, and experts in this field have focused on interdisciplinary distinctions and have considered a theoretical model necessary to build a more comprehensive and common view. Also, Kant (47), Habermas $(48,26)$ and Wilber $(49,26)$ argue the general epistemological structure of knowledge is based on more than 1 discipline and clearly point to the fact that the scientific disciplines have different descriptions of phenomena that are examined from the perspective of that discipline and disciplines should be interconnected to achieve a common core perspective.

Stephen Toulmin stated, "Interdisciplinary ideas have been built based on disciplines and interdisciplinary studies depend on them." (9)

According to Boix Mansilla, "interdisciplinary understanding greatly takes much of its information from disciplinary activities" $(7,10)$.

Chrysostomou (50) believes scientific disciplines play an important role in interdisciplinary formation. Before an interdisciplinary integration, a clear and precise framework of scientific discipline should exist and the structure of the scientific discipline be identified. Otherwise, the result would be a shallow knowledge and superficial and irregular thinking.

Fuchsman and Henry, Razzaq et al, Newell, and Moran $(4,7,51,52)$ argue interdisciplinary activities are clearly evolving from disciplines. The disciplines divide knowledge into separate units, while interdisciplinarity integrates these separate units. Interdisciplinary activities can open the closed boundaries of disciplines and allow a multidimensional view to complex problems of the real world.

By reviewing the literature based on disciplinary boundaries explained from the results in the first stage of the study, a multilayered model was presented for interdisciplinary development (Fig. 2).

It seems that a multilayered model is a suitable model for illustrating the relationship and integration of the boundaries of different disciplines in interdisciplinary activities. In this process, we will see differences in philosophy, culture, language, priorities, aim, knowledge, human resource, institution, resources, interactions, and economic and noneconomic values. Implementing interdisciplinary relationships and integration requires further study of the methods that establish relationships among boundaries. In the next section of study, we will examine the operationalization of this theoretical model in interdisciplinary activities to have a clear understanding of operationalizing interdisciplinary activities.

\section{Conclusion}

Interdisciplinary activities focus on integrating the boundaries of the scientific discipline to solve complex real-world problems. For interdisciplinarity, the artificial boundaries of the disciplines should be broken and opened; therefore, disciplinary specialists with the goal of solving complex social problems go beyond the disciplinary boundaries and reduce the distance or the displacement of the disciplinary boundaries.

Thus, we first began to explain the disciplinary boundaries in 13 levels, and then proposed a multilayered model of interdisciplinary development by examining the views of the experts in this field, and with the opinion and synthesis of researchers of this study on each of the disciplinary boundaries. It seems this model can effectively support and bring the goals of interdisciplinary programs closer to practical reality.

\section{Acknowledgement}

This article was part of a PhD dissertation in medical education conducted at Shahid Beheshti University of Medical Sciences. The authors would like to thank Ebrahim Saadatju for searching publications.

\section{Conflict of Interests}

The authors declare that they have no competing interests.

\section{References}

1. Meleis AI. Theoretical nursing: Development and progress. $5^{\text {th }} \mathrm{ed}$. Philadelphia, PA: Lippincott Williams \& Wilkins; 2011.

2. Aboelela SW, Larson E, Bakken S, Carrasquillo O, Formicola A, Glied SA, et al. Defining interdisciplinary research: conclusions from a critical review of the literature. Health Serv Res. 2007;42:329-46.

3. Pirrie A, Wilson V, Elsegood J, Hall J, Hamilton S, Harden R, et al. Evaluating Multidisciplinary Education In Health Care. United Kingdom: Eric; 1998.

4. Razzaq J, Townsend T, Pisapia J. Towards An Understanding of Interdisciplinarity: The Case Of A British University. Issu Interdiscip Stud. 2013;31:149-73.

5. Rogers Y, Scaife M, Rizzo A. Interdisciplinarity: an Emergent or Engineered Process? Interdiscip Collab Emerg Cognit Sci. 2005;26586.

6. Kockelmans JJ. Why interdisciplinarity. In: Kockelmans JJ, editor. Interdisciplinarity and higher education. Pennsylvania: Penn State Press; 1979. pp. 123-160. 
7. Fuchsman K, Henry S. Rethinking integration in interdisciplinary studies. Issu Interdiscip Stud. 2009;27:70-85.

8. Carnwell R, Daly W. Strategies for the construction of a critical review of the literature. Nurse Educ Pract. 2001;1(2):57-63.

9. Toulmin SE. Return to reason. Cambridge, MA: Harvard University Press; 2009.

10. Mansilla VB. Assessing Student Work at Disciplinary Crossroads. Chang Mag High Learn. 2005;37(1):14-21.

11. Gabbay D, Thagard P, Woods J, Kuipers T. General philosophy of science: Focal issues. Amsterdam: Elsevier; 2007.

12. Rousseau D, Wilby J, Billingham J, Blachfellner S. The scope and range of general systems transdisciplinarity. Syst Connect Matter Life Cult Technol. 2016;4, 48-60.

13. Schmidt JC. Towards a philosophy of interdisciplinarity. Poiesis Prax. 2008;5(1):53-69.

14. Little D. Beyond Positivism: Toward a Methodological Pluralism for the Social Sciences. Michigan: University of Michigan; 2004. Available from: http://www.personal.umd.umich.edu/ delittle/ BEYPOSIT.PDF.

15. Miller TR, Baird TD, Littlefield CM, Kofinas G, Chapin F, Redman CL. Epistemological pluralism: reorganizing interdisciplinary research. Ecol Soc. 2008;13(2): 46.

16. Miller M, Mansilla V. Thinking Across Perspectives and Disciplines. Boston, MA: Harvard Graduate School of Education; 2004.

17. Ylijoki O-H. Disciplinary cultures and the moral order of studying A case-study of four Finnish university departments. High Educ. 2000;39(3):339-62.

18. Wesselink A. Interdisciplinarity, problem focused research and normativity. SRI Papers. 2008;11:1-20.

19. Klein JT. Enhancing Communication \& Collaboration in Interdisciplinary Research. Thousand Oaks, California: SAGE Publications; 2013. pp. 11-30. Availabe from: http://methods. sagepub.com/book/enhancing-communication-collaboration-idresearch.

20. Wear DN. Challenges to interdisciplinary discourse. Ecosystems. 1999;2:299-301.

21. McCallin AM. Pluralistic Dialogue: A Grounded Theory of Interdisciplinary Practice. Aust J Rehabilitation Couns. 1999;5(2):7885 .

22. Monteiro M, Keating E. Managing misunderstandings: The role of language in interdisciplinary scientific collaboration. Sci Commun. 2009;31:6-28

23. Schnieder L, Wegener M. Transgression of semantic boundaries by methodical terminology management. Leicester: International Symposium on Spatial Accuracy Assessment. 2010;9.

24. Vosskamp W, Miller RC, Klein JT. From scientific specialization to the dialogue between the disciplines. Issu Interdiscip Stud. 1986;4:1736.

25. Fischer KW. A theory of cognitive development: The control and construction of hierarchies of skills. Psychol Rev. 1980;87(6):477531.

26. Stein Z. Modeling the demands of interdisciplinarity: Toward a framework for evaluating interdisciplinary endeavors. Integr Rev. 2007;4:91-107.

27. Besselaar VD, Heimeriks G. Disciplinary, multidisciplinary, interdisciplinary: Concepts and indicators. Paper for the 8th conference on Scientometrics and Informetrics - ISSI 2001 Sydney. Australia, July 16-20, 2001.

28. Sawa RJ. Foundations of interdisciplinarity: a Lonergan perspective. Med Health Care Philos. 2005;8(1):53-61.

29. Aram JD. Concepts of Interdisciplinarity: Configurations of Knowledge and Action. Hum Relat. 2004;57(4):379-412.

30. MILLAR, V. 2016. Interdisciplinary curriculum reform in the changing university. Teaching in Higher Education, 21, 471-483.

31. Lattuca LR. Creating interdisciplinarity: Grounded definitions from college and university faculty. Hist Intellect Cult. 2003;3:78-119.

32. Jakab E, Lukic J. Disciplinization: Process And Problematics. A comparative of eight European countries. Research Integration. 2005:1-50. Available from: https://www.york.ac.uk/res/research integration/ComparativeReports/Comparative_Report_Disciplinizatio n.pdf

33. Palmer MA, Kramer JG, Boyd J, Hawthorne D. Practices for facilitating interdisciplinary synthetic research: the National SocioEnvironmental Synthesis Center (SESYNC). Curr Opin Environ Sustain. 2016;19:111-22.
34. Klein JT. Finding interdisciplinary knowledge and information. N Dir Teach Learn. 1994;58:7-33.

35. Berte M. Transdisciplinarity and Education:"The treasure within"Towards a transdisciplinary evolution of education. Brazil: The 2nd World Congress of Transdisciplinarity, 2005.

36. Prager K, Morris S, Currie M, Macleod K. Exploring Interdisciplinarity: summary report of the 'Developing an interdisciplinary culture of excellence (DICE)'project at the James Hutton Institute. Scotland, UK: The James Hutton Institute; 2015.

37. Burger P, Kamber R. Cognitive integration in transdisciplinary science: Knowledge as a key notion. Issu Interdiscip Stud. 2003;21:43-73.

38. Jacobs JA, Frickel S. Interdisciplinarity: A Critical Assessment. Ann Rev Sociol. 2009;35(1):43-65.

39. O'rourke M, Crowley S, Eigenbrode SD, Wulfhorst JD. Enhancing communication \& collaboration in interdisciplinary research. Thousand Oaks, California: Sage Publications; 2013.

40. Johnson AG. "Deep Interdisciplinarity" as Critical Pedagogy: Teaching at the Intersections of Urban Communication and Public Place and Space. Int J Crit Pedag. 2014;5(2):5-23.

41. Klein JT. Interdisciplinarity: History, Theory, and Practice. Midtown Detroit: Wayne State University Press; 1990.

42. FROST SH, Jean PM. Bridging the disciplines: Interdisciplinary discourse and faculty scholarship. J High Educ. 2003;74:119-149.

43. Jean-Claude A, Frochot C. Problems arising in evaluation of interdisciplinary scientific research for innovation. Int $\mathrm{J}$ Innov Sci. 2013;5(2):103-12.

44. Holley KA. Understanding interdisciplinary challenges and opportunities in higher education. Hoboken, New Jersey: Wiley Jossey-Bass; 2009.

45. Lyall C, Bruce A, Marsden W, Meagher L. Key success factors in the quest for interdisciplinary knowledge. Report to NERC; 2011. Available from: https://core.ac.uk/download/pdf/28965045.pdf

46. Nowacek RS. A discourse-based theory of interdisciplinary connections. J Gen Educ. 2005;54(3):171-95.

47. Kant 1998 KANT, I. 1998. Critique of Pure Reason (translated and edited by Paul Guyer \& Allen W. Wood). A 550/N 578.

48. Habermas J. Moral consciousness and communicative action. Cambridge, Massachusetts: MIT press; 1990.

49. Wilber K. Integral spirituality. Boston, MA: Integral Book: 2006.

50. Chrysostomou S. Interdisciplinary approaches in the new curriculum in Greece: A focus on music education. Arts Educ Policy Rev. 2004; 105:23-30.

51. Newell WH. Six arguments for agreeing on a definition of interdisciplinary studies. AIS newsl. 2007;29:1-4.

52. Moran J. Interdisciplinarity. Abingdon-on-Thame: Routledge; 2010. 